Research article

Open Access

\title{
Differential expression of the FAK family kinases in rheumatoid arthritis and osteoarthritis synovial tissues
}

\author{
Shiva Shahrara ${ }^{1}$, Hernan P Castro-Rueda1 ${ }^{1}$ G Kenneth Haines ${ }^{2,3}$ and Alisa E Koch ${ }^{4,5,1}$
}

\author{
1Department of Medicine, Feinberg School of Medicine, Northwestern University, Chicago, Illinois 60611, USA \\ 2Department of Pathology, Feinberg School of Medicine, Northwestern University, Chicago, Illinois 60611, USA \\ ${ }^{3}$ Department of Pathology, Yale University School of Medicine, New Haven, Connecticut 06511, USA \\ ${ }^{4}$ Veteran's Administration, Chicago Health Care System, Lakeside Division, Chicago Illinois 60611, USA \\ ${ }^{5}$ Veteran's Administration and the University of Michigan Medical Center, Ann Arbor, Michigan 48109, USA
}

Corresponding author: Alisa E Koch, aekoch@med.umich.edu

Received: 8 May 2007 Revisions requested: 19 Jun 2007 Revisions received: 9 Oct 2007 Accepted: 26 Oct 2007 Published: 26 Oct 2007

Arthritis Research \& Therapy 2007, 9:R112 (doi:10.1186/ar2318)

This article is online at: http://arthritis-research.com/content/9/5/R112

(c) 2007 Koch et al.; licensee BioMed Central Ltd.

This is an open access article distributed under the terms of the Creative Commons Attribution License (http://creativecommons.org/licenses/by/2.0), which permits unrestricted use, distribution, and reproduction in any medium, provided the original work is properly cited.

\begin{abstract}
The focal adhesion kinase (FAK) family kinases, including FAK and proline-rich kinase 2 (Pyk)2, are the predominant mediators of integrin $\alpha v \beta 3$ signaling events that play an important role in cell adhesion, osteoclast pathology, and angiogenesis, all processes important in rheumatoid arthritis (RA). Using immunohistochemical and western blot analysis, we studied the distribution of phospho (p)FAK, pPyk2, pSrc, pPaxillin and pPLC $\gamma$ in the synovial tissue (ST) from patients with RA, osteoarthritis (OA) and normal donors (NDs) as well as in RA ST fibroblasts and peripheral blood differentiated macrophages (PB MФs) treated with tumor necrosis factor- $\alpha$ (TNF $\alpha$ ) or interleukin-1 $\beta$ (IL1 $\beta)$. RA and OA STs showed a greater percentage of pFAK on lining cells and MФs compared with ND
\end{abstract}

ST. RA ST fibroblasts expressed pFAK at baseline, which increased with TNF $\alpha$ or IL1 $\beta$ stimulation. Pyk2 and Src were phosphorylated more on RA versus OA and ND lining cells and MФs. pPyk2 was expressed on RA ST fibrobasts but not in MФs at baseline, however it was upregulated upon TNF $\alpha$ or IL1 $\beta$ activation in both cell types. pSrc was expressed in RA ST fibroblasts and MФs at baseline and was further increased by TNF $\alpha$ or IL $1 \beta$ stimulation. pPaxillin and pPLC $\gamma$ were upregulated in RA versus OA and ND lining cells and sublining MФs. Activation of the FAK family signaling cascade on RA and OA lining cells may be responsible for cell adhesion and migration into the diseased STs. Therapies targeting this novel signaling pathway may be beneficial in RA.

\section{Introduction}

In rheumatoid arthritis (RA), macrophages (MФs) derived from circulating monocytes are key regulators of joint inflammation and destruction. Hence, suppression of cell adhesion and migration into the RA synovial tissue (ST) may ameliorate inflammation. In this study we determined integrin-associated signaling molecules that become activated, probably as a result of inflammation in RA ST. Focal adhesion kinase (FAK) and proline-rich tyrosine kinase (Pyk)2 are two members of a family of nonreceptor protein tyrosine kinases that are activated by a variety of extracellular stimuli [1]. FAK and Pyk2 associate with the cytoskeleton and with integrin-signaling complexes by binding to Src kinase and paxillin [2-5]. FAK is rapidly tyrosine phosphorylated on cell adhesion, creating a high-affinity binding site for Src and thereby increasing phospholipase C (PLC) $\gamma$ enzymatic activity [6]. Paxillin is a substrate for the FAK-Src complex that functions as an adaptor molecule for various signaling and structural proteins, and can promote migration of fibroblasts, MФs and endothelial cells [7$11]$.

FAK expression is ubiquitous and FAK is activated by numerous integrins, suggesting that FAK activation is common adhesion-dependent signal [12-14]. Unlike FAK, Pyk2 expression is highly cell-type and tissue specific. Pyk2 is tyrosine phosphorylated in response to stress (UV irradiation, tumor necrosis factor- $\alpha$ (TNF $\alpha)$ and hyperosmotic shock), G protein-coupled receptor agonists (angiotensin II, thrombin) and growth factors (vascular endothelial growth factor (VEGF), basic fibroblast growth factor (bFGF), and platelet derived growth factor (PDGF) [15-17]. Although FAK activation is closely tied to integrin-mediated adhesion, activation of Pyk2 can be independent of cell adhesion [18]. FAK and Pyk2 are expressed in osteoclasts, and both proteins are tyrosine phosphorylated in response to integrin $\alpha v \beta 3$ ligation, a process which may be crucial for bone resorption $[3,19]$. Both FAK and Pyk2 play a 
central role in linking integrin $\alpha v \beta 3$ signaling to the formation of podosomes and actin rings in osteoclasts. Although FAK is phosphorylated by Src, Pyk2 can be phosphorylated through a Src- or a $\mathrm{Ca}^{2+-d e p e n d e n t ~ p a t h w a y ~[18] . ~ A d d i t i o n a l l y, ~ F A K ~ i s ~}$ involved in angiopoietin-1 and VEGF-induced endothelial cell migration and angiogenesis $[8,20]$; however, the role of Pyk2 in endothelial cell function has not been explored.

MФs isolated from RA ST have the potential to differentiate to osteoclasts in the presence of receptor activator of NF-kappaB ligand (RANKL) and macrophage colony stimulating factor (M-CSF) [21]. Stimulation of PB monocytes with M-CSF mediates FAK activation, suggesting that FAK may be involved in monocyte differentiation into MФs [22]. Interestingly, in rat adjuvant induced arthritis (AIA) intra-articular injection of dominant negative FAK adenovirus reduces mononuclear cell recruitment into the joint. Inhibition of FAK suppresses VEGFinduced mononuclear cell migration into the AIA ankle [8]. This suggests that suppression of FAK activation may be important for reducing cell recruitment into RA ST.

In this study we investigated the expression pattern of pFAK, pPyk2, pSrc, pPaxillin and pPLC $\gamma$ in RA and OA ST. Activation of these signaling proteins on RA and OA ST lining cells may be responsible for monocyte adhesion and migration into the diseased STs, whereas activation of these signaling proteins on MФs may be important for both monocyte to MФ differentiation as well as $\mathrm{M} \Phi$ differentiation into osteoclasts.

\section{Materials and methods}

STs were obtained from patients diagnosed with RA and OA undergoing arthroplasty or synovectomy. RA or OA were diagnosed according to the criteria of the American Collage of Rheumatology $[23,24]$. Normal STs, were obtained from fresh autopsies or amputations. STs, were snap frozen in OCT compound (Miles, Elkhart, Indiana, USA). All samples were obtained with Institutional Review Board approval additionally informed patient consent or consent from next of kin was documented.

\section{Antibodies and immunohistochemistry}

STs were cut into $4 \mu \mathrm{m}$ sections and fixed in cold acetone for 20 minutes. Endogenous peroxidase was quenched by treatment with $3 \% \mathrm{H}_{2} \mathrm{O}_{2}$ for 5 minutes. STs were next pretreated with $3 \%$ goat sera for 1 hour at $37^{\circ} \mathrm{C}$ before application of primary antibody in $4^{\circ} \mathrm{C}$ overnight. Indirect immunoperoxidase staining was performed at $37^{\circ} \mathrm{C}$ for 1 hour. Polyclonal antibody $(\mathrm{pAb})$ rabbit anti-human $\mathrm{pFAK}, \mathrm{pAb}$ rabbit anti-human pPyk2, pAb rabbit anti-human pSrc, pAb rabbit anti-human $\mathrm{pPaxillin}$ and $\mathrm{pAb}$ rabbit anti-human $\mathrm{pPLC} \gamma$ were all purchased from Biosource (Camarillo, California, USA) or Cell Signaling Technology (Beverly, Massachusetts, USA), and were used at a concentration of $1 \mu \mathrm{g} / \mathrm{ml}$. Isotype-specific lgG (rabbit) was used as a negative control. Staining was performed using Vector Elite ABC Kits (Vector, Burlingame, California, USA) and diaminobenzidine (Kirkegaard and Perry, Gaithersburg, Maryland, USA) as a chromogen.

\section{Microscopic analysis}

Vascularity was defined as a score as follows: 1, marked decrease in vessels; 2 , normal density of vessels; 3 , increased density of vessels; 4, marked increase in vessel density, resembling granulation tissue. Inflammation was defined as a score as follows: 1, normal; 2, mildly increased number of inflammatory cells, arrayed as individual cells; 3, moderately increased number of inflammatory cells including distinct clusters (aggregates); 4, marked diffuse infiltrate of inflammatory cells. MФs were distinguished from fibroblasts based on morphology and CD 11b/c immunoreactivity. Score data were pooled and the mean \pm SEM was calculated in each data group [25-27]. Each of the ST components was graded for immunostaining by a frequency of attaining scale, scored 0$100 \%$ where $0 \%$ indicates no staining and $100 \%$ indicates that all cells were immunoreactive. The number of cells of a given type that reacted with a specific antibody divided by the total number of cells of that given type was defined as the percentage of reactivity. The mean percentage of reactivity was determined for 3 high power fields (HPF) in STs for each cell type and antibody analyzed. Each slide was evaluated by a single blinded pathologist (GKH). Selected sections were analyzed by an additional observer (SS).

\section{Cell culture and western blot analysis}

RA fibroblasts were isolated from fresh STs by mincing and digesting in a solution of dispase, collagenase and DNase [28]. Cells were used at passage 4 or older, at which time they are a homogeneous population of fibroblasts. Cells were cultured in DMEM containing 10\% heat-inactivated fetal bovine serum (FBS) [29]. Mononuclear cells were isolated by Histopaque (Sigma Chemical Co., St. Louis, Missouri, USA) gradient centrifugation. PB monocytes were then isolated from the mononuclear cells by Percoll (Sigma Chemical Co.) gradient centrifugation and countercurrent centrifugal elutriation (Beckman-Coulter, Fullerton, California, USA) [29]. Following adherence, monocytes were differentiated in vitro for 7 days in RPMI containing 20\% FBS plus $1 \mu \mathrm{g} / \mathrm{ml}$ polymyxin B sulfate (Sigma Chemical Co). As PB monocytes were isolated from buffy coats by elutriation polymyxin $B$ was added to the media preventatively. The endotoxin levels in RPMI, FBS and PBS as measured by Limulus Amebocyte Lysate (LAL) (QCL-1000; Cambrex Bioscience, Maryland, USA) were below the lowest detectable level of 0.1 endotoxin unit (EU). The endotoxin levels in TNF $\alpha$ and IL1 $\beta$ were lower than $1.0 \mathrm{EU}$ per $1 \mu \mathrm{g}$ of the cytokine as determined by the LAL method.

RA ST fibroblasts (cultured in DMEM with 10\% FBS) and MФs (cultured in RPMI with 20\% FBS) were either untreated or treated with TNF $\alpha$ (10 ng/ml; R\&D Systems, Minneapolis, New Mexico, USA) [30] or IL1 $\beta$ (10 ng/ml; R\&D Systems) [31] for 0 to $120 \mathrm{~min}$. 
Western blot analysis was conducted as previously described [29]. Briefly, $60 \mu \mathrm{g}$ of each sample was loaded on a $10 \%$ SDS-PAGE gel and transferred to nitrocellulose membranes using a semi-dry transblotting apparatus (Bio-Rad, Hercules, California, USA). Nitrocellulose membranes were blocked with $5 \%$ nonfat milk in Tris-buffered saline Tween (TBST) buffer-20 $\mathrm{mM}$ Tris, $137 \mathrm{mM} \mathrm{NaCl}, \mathrm{pH} 7.6$, with $0.1 \%$ Tween for $60 \mathrm{~min}$ at room temperature. Blots were probed with rabbit anti-pFAK (Tyr 576/577), anti-pPyk2 (Tyr 402), or anti-pSrc (Tyr 527) (Cell Signaling Technology) overnight and after stripping reprobed with rabbit anti-FAK, anti-Pyk2 or anti-Src (Cell Signaling Technology at 1:1000) overnight.

\section{Statistical analysis}

The data was analyzed using Student's $t$-tests. $P$ values less than 0.05 were considered significant.

\section{Results pFAK localization}

As expected, the inflammatory and vascularity scores were higher in RA ST in comparison to OA and NDs. pFAK, was expressed on ST lining cells in RA patients (mean of $15 \%$ pos- itive cells) and OA (21\%) more than on ND ST lining (1\%) $(P$ $=<0.05$ ) (Figure 1). pFAK staining on MФs was also significantly higher in RA (39\%) and OA (25\%) compared to ND $(4 \%)(P<0.05)$. A few RA patients had positive immunostaining for pFAK on ST endothelial cells and lymphocytes. Unstimulated RA ST fibroblasts expressed pFAK; however, the expression increased with TNF $\alpha$ stimulation at $45 \mathrm{~min}$ and stayed upregulated until 120 min (Figure 1e). Similarly, IL1 $\beta$ increased pFAK expression at 30, 45 and 120 min in RA ST fibroblasts (Figure 1f). pFAK was not detected in MФs with or without TNF $\alpha$ or IL1 $\beta$ stimulation.

\section{pPyk2 expression pattern}

pPyk2 is one of the members of the nonreceptor protein tyrosine kinase FAK family and shares approximately 45\% sequence homology with FAK. Both proteins are important for integrin-mediated adhesion and osteoclastogenesis [18]. pPyk2 immunostaining on ST lining and MФs was significantly higher in RA (lining cells = 60\% and MФs = 46\%) compared to OA (lining cells $=30 \%$ and $\mathrm{M \Phi s}=23 \%$ ) and ND (lining cells $=17 \%$ and $M \Phi s=10 \%)(P<0.05)$ (Figure 2). However, no difference was detected in pPyk2 lining cells and $М \Phi$

Figure 1

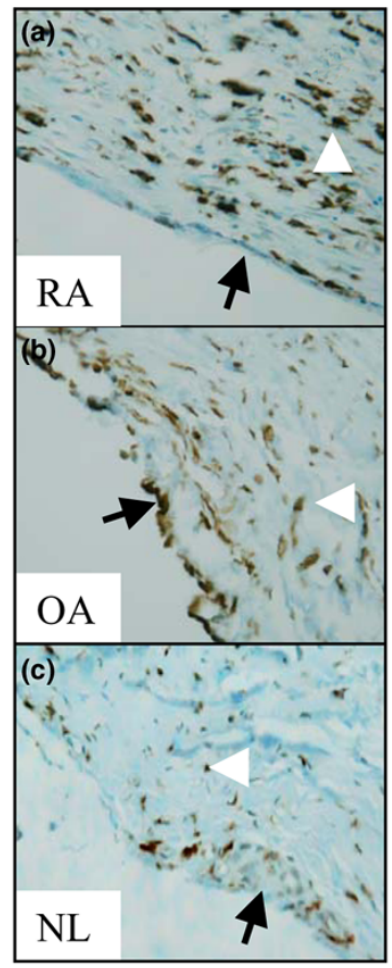

(d)

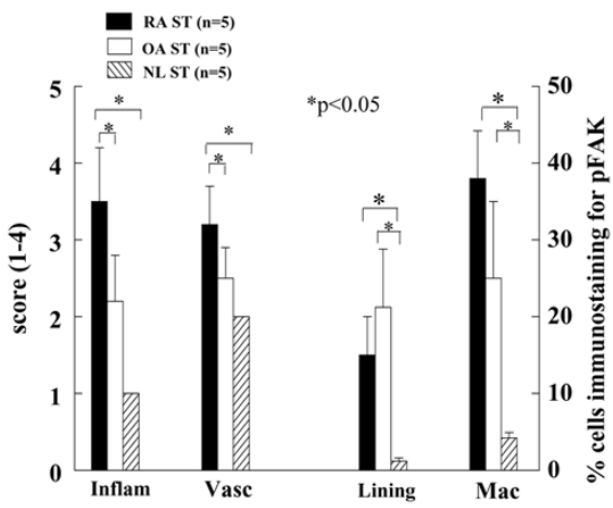

(e)
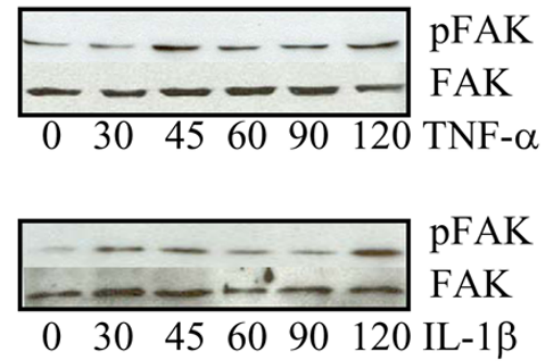

pFAK expression pattern in RA, OA and normal donor (ND) ST. (a) Rheumatoid arthritis synovial tissue (RA ST) stained with anti-pFAK, showing positive staining of the lining cell layer (black arrow) and subsynovial MФs (white arrowhead) ( $\times 200)$. (b) Positive staining in osteoarthritis (OA) ST ( $\times 200)$. (c) Low pFAK reactivity in normal ST lining (arrow) and subsynovial macrophages (arrowhead). (d) The quantification of data obtained from $\mathrm{a}, \mathrm{b}$ and c. Bars represent the mean and SEM. Inflam, inflammatory score; Vasc, vascularity score; Lining, ST lining cell layer; Mac, subsynovial MФs. ${ }^{*} P<0.05$. $\mathrm{n}$, numbers of patients. RA ST fibroblasts were stimulated with (e) tumor necrosis factor- $\alpha$ (TNF- $\left.\alpha\right)(10 \mathrm{ng} / \mathrm{ml})$ or $(\mathrm{f})$ interleukin- $1 \beta(\mathrm{IL}-1 \beta)$ $(10 \mathrm{ng} / \mathrm{ml})$ from 0-120 min. Cell lysates were examined by western blot analysis for pFAK or FAK expression. The results are representative of three experiments. 

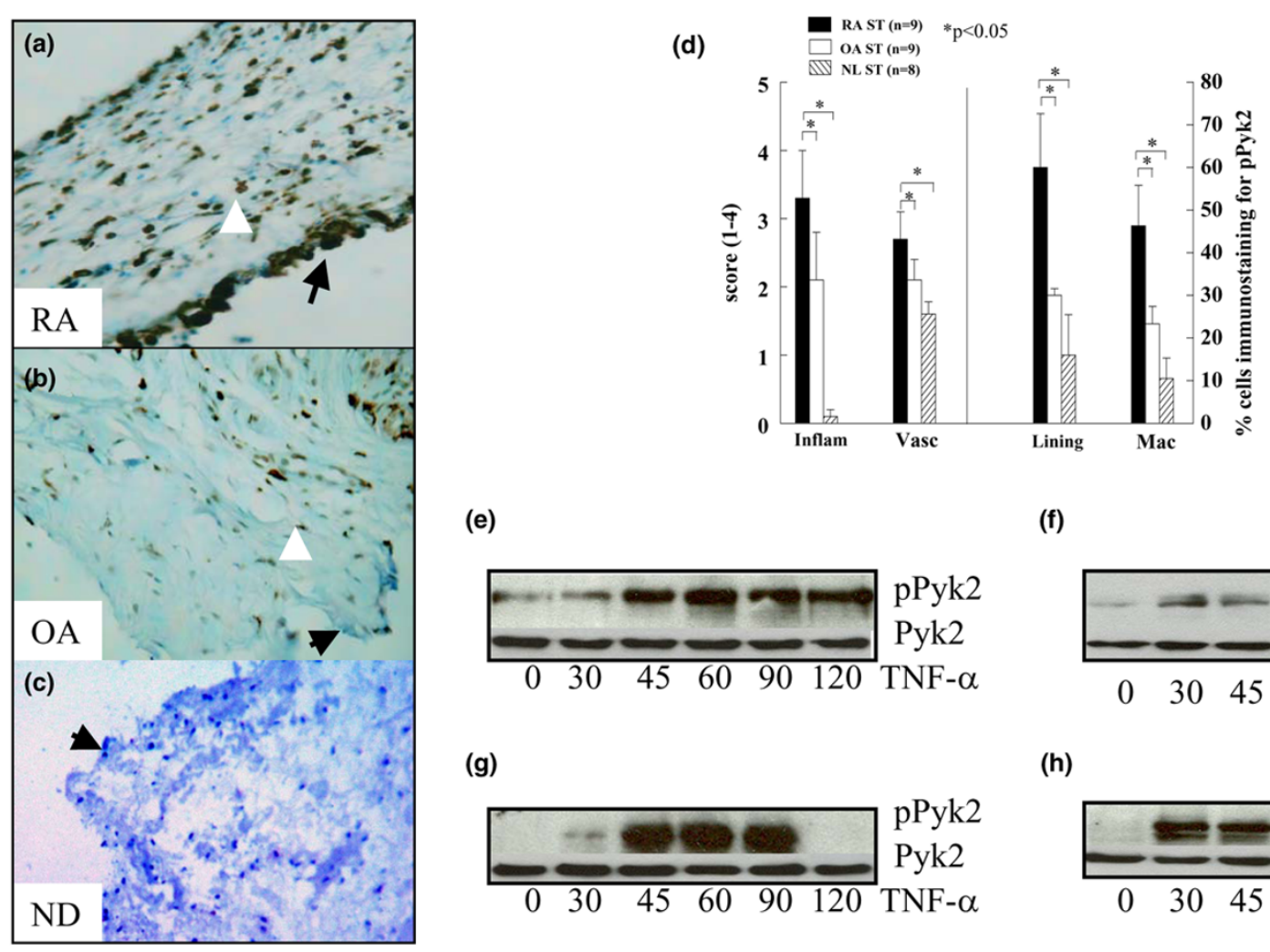

(e)

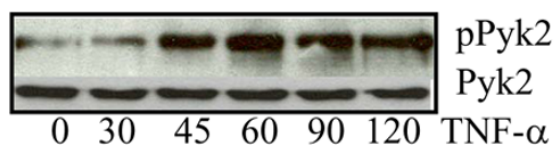

(g)

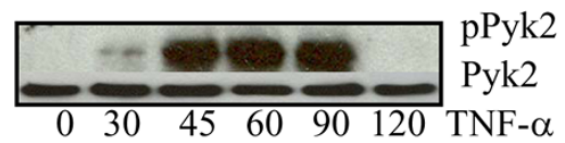

(f)

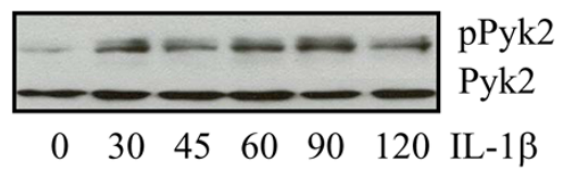

(h)

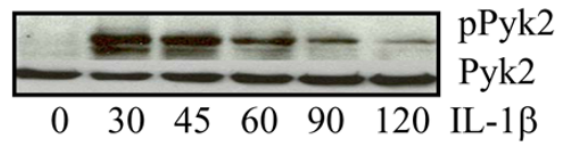

Rheumatoid arthritis synovial tissue (RA ST) had higher pPyk2 immunopositive cells compared to osteoarthritis (OA) ST. (a) RA ( $\times 200)$, compared to (b) OA ( $\times 200)$ and (c) normal donor (ND) (×200). (d) is the quantification data obtained from figure a and b. Bars represent mean and SEM. RA ST fibroblasts (e and $\mathbf{f})$ or peripheral blood differentiated MФs $(\mathbf{g}$ and $\mathbf{h})$ were stimulated with TNF- $\alpha(10 \mathrm{ng} / \mathrm{ml})$ or IL $1-\beta$ (10 ng/ml) from 0-120 min. Cell lysates were examined by western blot analysis for pPyk2 or Pyk2 expression. The results are representative of three experiments. Inflam, inflammatory score; Vasc, vascularity score; Lining, ST lining cell layer; Mac, subsynovial МФs.

immunostaining in OA and ND STs. The relative pattern of pFAK and pPyk2 expression was similar in RA patient MФs (pFAK $=39 \%$, pPyk2 = 46\%); however, pPyk2 was highly expressed on synovial lining ( $\mathrm{pFAK}=15 \%$, pPyk2 $=60 \%$ ) compared to pFAK. The relative pattern of pFAK and pPyk2 expression was similar in OA patients' synovial lining (pFAK = $21 \%$, pPyk2 = 30\%) and MФs (pFAK = 25\%, pPyk2 = 23\%). Interestingly, although pFAK was similarly expressed in RA and OA patients, the percentage of pPyk2 positive cells was significantly higher in RA ST lining and sublining compared to that of OA and ND. Rarely, RA patients showed positive immunostaining for pPyk2 on ST endothelial cells, fibroblasts and lymphocytes. pPyk2 was detected on unstimulated ST fibroblasts, and the expression was further increased by TNF $\alpha$ and IL $1 \beta$ stimulation and stayed upregulated up to $120 \mathrm{~min}$ (Figure 2d,e). MФs did not express pPyk2 at baseline; however, after 30 to 45 min stimulation with TNF $\alpha$ or IL1 $\beta$ a robust level of pPyk2 was detected. In MФs, pPyk2 remained activated for 90 min subsequent to TNF $\alpha$ or IL1 $\beta$ activation and thereafter markedly decreased (Figure $2 \mathrm{f}, \mathrm{h}$ ).

\section{pSrc localization}

Integrin $\alpha v \beta 3$ activation induces FAK and Pyk2 phosphorylation via Src. Phosphorylation of FAK and Pyk2 results in formation of a signaling complex consisting of signaling molecules including Src and paxillin. Formation of a Pyk2-Src complex and the kinase activity of Src is required for bone resorption by osteoclasts [32]. Src is highly phosphorylated in RA ST lining cells $(R A=68 \%)$ and MФs (RA $=57 \%)$ compared to OA (lining cells $=13 \%, M \Phi s=16 \%$ ) and ND ST (lining cells $=6 \%$, MФs $=10 \%$ ) (Figure 3). Although pSrc associated with both FAK and Pyk2 signaling complexes, the expression pattern of pSrc in RA ST lining (pSrc =68\%, pPyk2 =60\%) and sublining ( $\mathrm{pSrc}=57 \%$, pPyk2 $=46 \%$ ) was similar to pPyk2, while pSrc immunostaining in OA ST lining $(\mathrm{pSrc}=12 \%, \mathrm{pPyk} 2=$ $30 \%, \mathrm{pFAK}=21 \%)$ and sublining (pSrc $=15 \%, \mathrm{pPyk} 2=$ $23 \%$, pFAK $=25 \%$ ) was comparable to both Pyk2 and FAK. RA ST endothelial cells and lymphocytes were occasionally immunopositive for pSrc. Both RA ST fibroblasts and differentiated MФs expressed pSrc at baseline. pSrc remained activated in RA ST fibroblasts stimulated with TNF $\alpha$ up to 120 

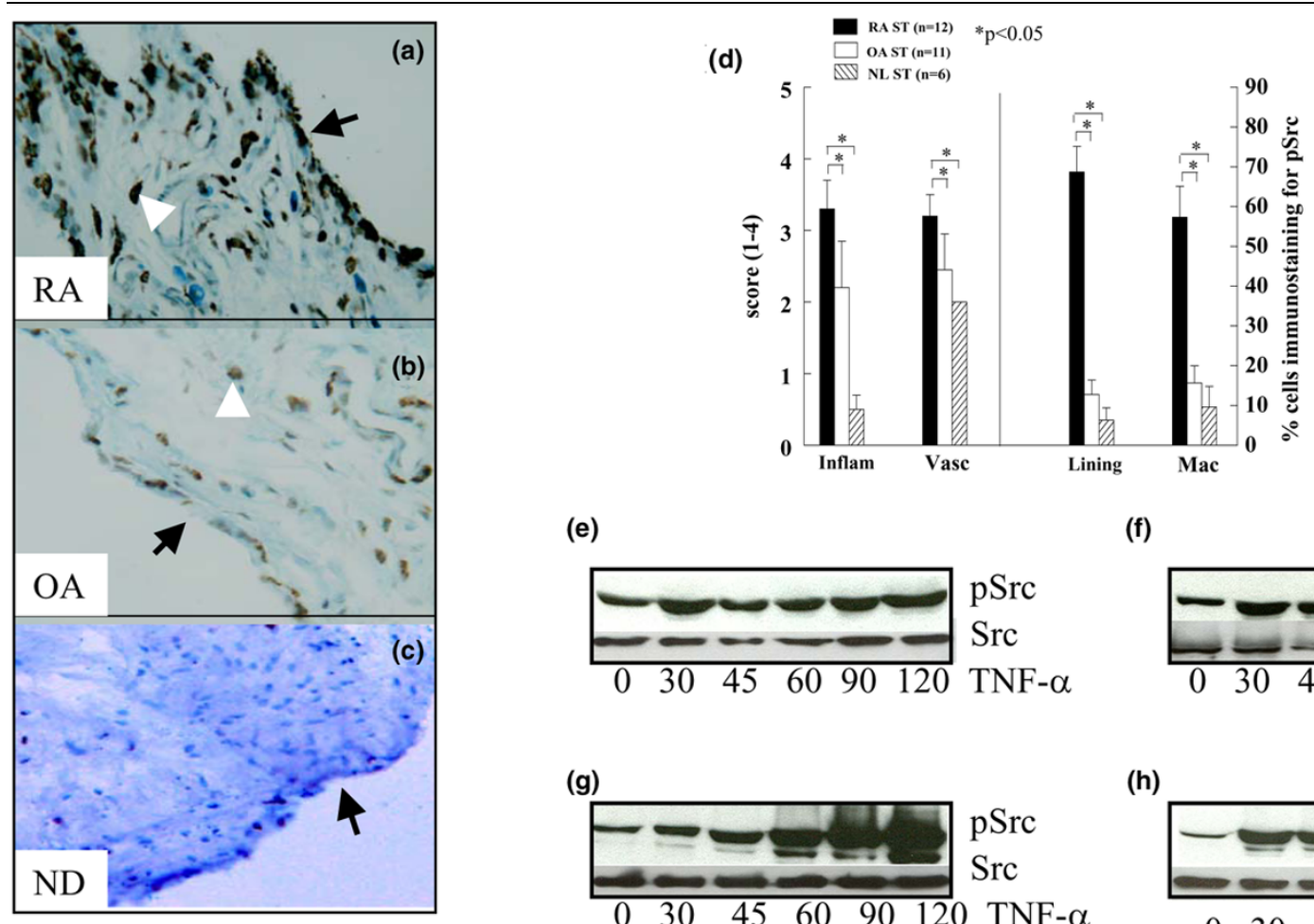

(e)

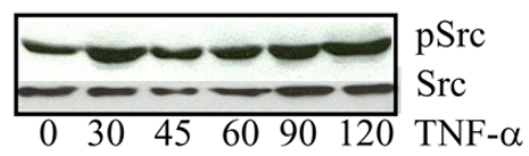

(g)

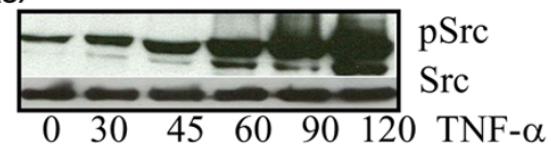

(f)

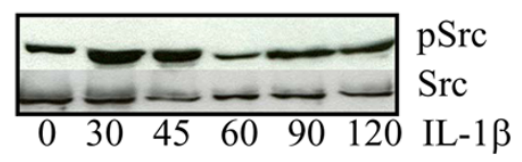

(h)

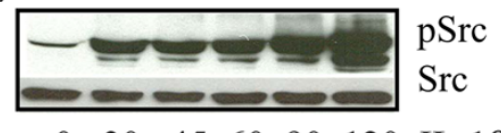

pSrc is upregulated in rheumatoid arthritis synovial tissue (RA ST) compared to osteoarthritis (OA) ST. (a) RA ( $\times 200)$, compared to (b) OA ( $\times 200)$ and (c) normal donor (ND) ( $\times 200)$. (d) is the quantification data obtained from a and b. Bars represent mean and SEM. RA ST fibroblasts (e and f) or peripheral blood differentiated MФs (g and $h$ ) were stimulated with TNF- $\alpha$ (10 ng/ml) or IL-1 $\beta$ (10 ng/ml) from 0-120 min. Cell lysates were examined by western blot analysis for pSrc or S Src expression. The results are representative of three experiments. Inflam, inflammatory score; Vasc, vascularity score; Lining, ST lining cell layer; Mac, subsynovial MФs.

min (Figure $3 d$ ). In contrast, IL $1 \beta$ induced activation of pSrc no longer than $45 \mathrm{~min}$ in RA ST fibroblasts (Figure 3e). In MФs, both TNF $\alpha$ and IL1 $\beta$ mediated robust activation of pSrc in a time dependent manner up to $120 \mathrm{~min}$ (Figure $3 \mathrm{f}$ and $3 \mathrm{~h}$ ).

\section{pPaxillin immunostaining}

Paxillin is a multidomain adaptor protein that interacts with signaling proteins such as FAK, Pyk2, Src and PLC $\gamma[6,33]$. The phosphorylation of paxillin is modulated by cell adhesion. Paxillin is recruited to the FAK and Pyk2 signaling complex upon integrin $\alpha v \beta 3$ ligation in osteoclasts. We found that pPaxillin is expressed on ST lining cells in RA patients (77\%) to a significantly higher degree than OA (37\%) and ND (12\%) $(P<$ 0.05). pPaxillin immunostaining on $\mathrm{M} \Phi$ s was also significantly higher in RA (70\%) compared to OA (40\%) and ND (14\%) $(P$ $<0.05$ ) (Figure 4). Similar to $\mathrm{pSrc}$ and $\mathrm{pPyk} 2$, paxillin was highly phosphorylated in RA ST lining (pPaxillin $=77 \%$, pSrc $=68 \%$, pPyk2 $=60 \%$ ) and sublining (pPaxillin $=70 \%$, pSrc $=$ $57 \%$, pPyk2 = 46\%). In contrast, pPaxillin immunostaining on OA lining ( $p$ Paxillin $=37 \%$, pPyk2 $=30 \%$, pFAK $=21 \%$ ) and sublining ( $p$ Paxillin $=40 \%$, pPyk2 $=23 \%$, pFAK $=25 \%$ ) was comparable to $\mathrm{pFAK}$ and pPyk2. Our findings suggest that the colocalization and activation of FAK, Pyk2, Src and paxillin in RA and OA patient's ST lining and sublining may be important for integrin-mediated signaling.

\section{pPLC $\gamma$ expression pattern}

Upon $\alpha v \beta 3$ integrin-mediated adhesion, PLC $\gamma$ associates with the Pyk2 and FAK signaling complex [6,33]. M-CSF can also induce association of $\alpha v \beta 3$ integrins with PLC $\gamma$, PI3K and Pyk2 in a Src-independent manner [33]. The inflammatory and vascularity scores for $\mathrm{pPLC} \gamma$ immunostaining were higher in RA ST in comparison to OA and ND. RA patients most strongly expressed pPLC $\gamma$ in the lining (67\%) and on MФs $(61 \%)$ in ST, compared to OA patients (lining = 9\%, MФs = $28 \%$ ) and ND subjects (lining $=10 \%, M \Phi s=14 \%$ )(Figure 5). Interestingly pPLC $\gamma$ immunostaining was similar on OA and ND MФs. The positive immunostaining of pPLC $\gamma$ was comparable to pPyk2 expression on RA ST lining (pPLC $\gamma=67 \%$, pPyk2 $=60 \%)$ and sublining (pPLC $\gamma=61 \%$, pPyk2 $=46 \%$ ). Whereas, pPLC $\gamma$ immunostaining on OA lining (pPLC $\gamma=9 \%$, pPyk2 $=30 \%$ ) was lower than that of pPyk2. These results suggest that Pyk2 and its associated signaling protein complex, namely Src, paxillin, and PLC $\gamma$ are activated on the RA ST 


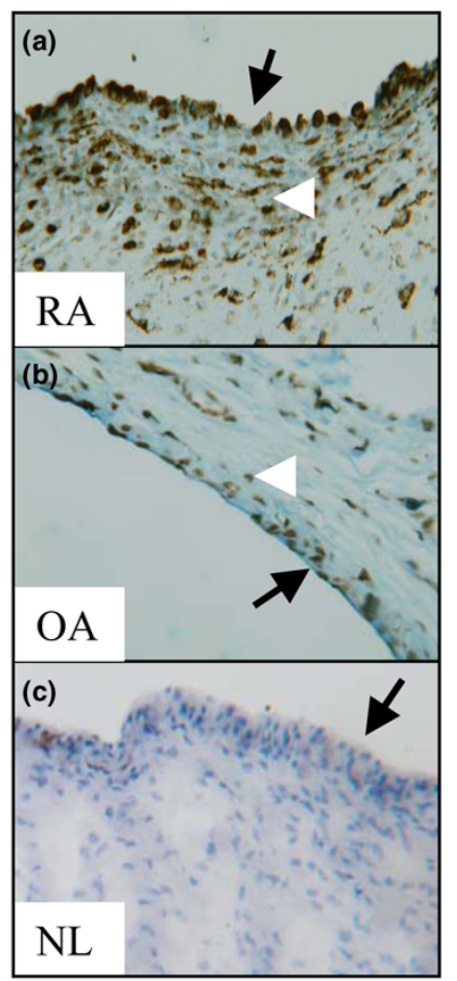

(d)

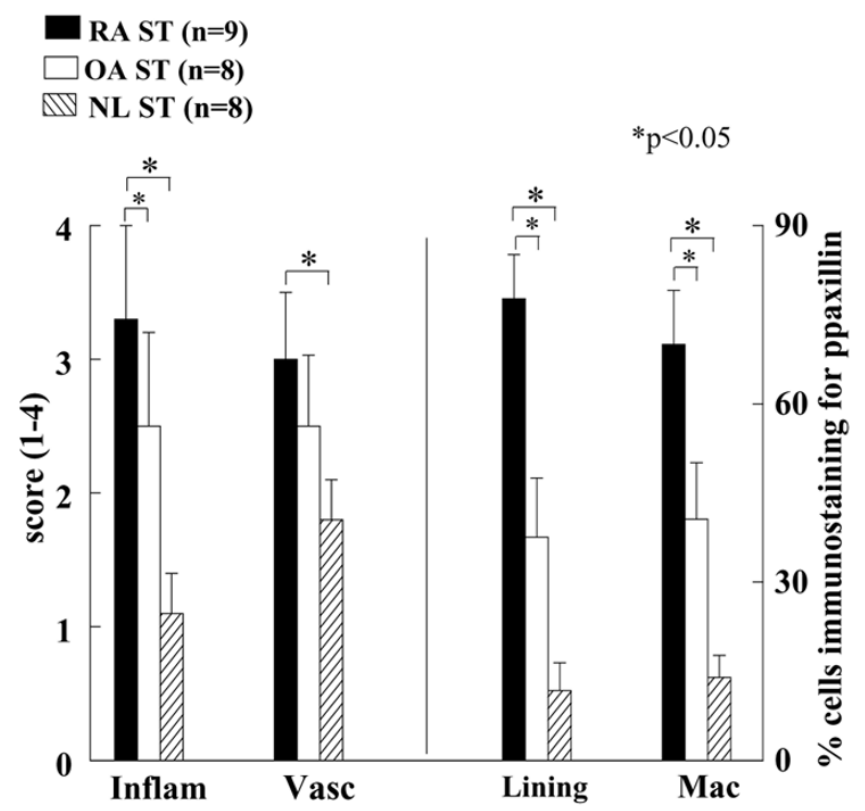

Immunostaining of pPaxillin is increased in rheumatoid arthritis synovial tissue (RA ST) compared to osteoarthritis (OA) and normal donor (ND) ST. (a) demonstrates RA ST stained with anti-pPaxillin $(\times 200)$, (b) shows positive staining in OA ST $(\times 200)$. (c) Low pPaxillin reactivity in normal ST lining and subsynovial MФs. (d) Is the quantification data obtained from a, b and c. Bars represent mean and SEM. Inflam, inflammatory score; Vasc, vascularity score; Lining, ST lining cell layer; Mac, subsynovial MФs.

lining and MФs to a greater extent than on OA ST. A few RA patients had positive immunostaining for PPLC $\gamma$ on fibroblasts and lymphocytes.

\section{Discussion}

RA is a chronic inflammatory disease characterized by synovial hyperplasia. Proliferation of synovial cells leads to pannus formation resulting in progressive bone and joint destruction. It has been reported that FAK and Pyk2 are involved in integrin $\alpha v \beta 3-m e d i a t e d$ bone resorption [19,34-36]. Interestingly little is known about the activation of these proteins in RA ST. In this study we demonstrated the phosophorylation of FAK and Pyk2 as well as their downstream signaling molecules, namely, Src, paxillin and PLC $\gamma$ in arthritic ST (Figure 6). In addition, we determined differences between diseased and ND STs in regards to these molecules.

Angiogenesis is important in the growth and proliferation of the RA ST pannus. FAK exerts its pro-angiogenic effects through multiple mechanisms. Angiopoietin-1 induced endothelial cell sprouting through FAK [37]. VEGF-mediated endothelial cell migration and tube formation occurred through FAK phosphorylation and subsequent PI3K activation [7]. Additionally, VEGF induced FAK tyrosine phosphorylation in RAW cells.
VEGF-mediated RAW cell chemotaxis was suppressed by dominant-negative FAK adenovirus [8]. In rat AIA, intra-articular injection of dominant negative adenoviral FAK reduced mononuclear cell recruitment into the joint [8]. Inhibition of FAK phosphorylation suppressed 3T3 fibroblast and human umbilical vein endothelial cell migration [9]. These results suggest that activation of the FAK signaling pathway may be important for fibroblast, macrophage and endothelial cell migration, all processes involved in RA ST inflammation and proliferation.

Upon localization to the integrin cluster, FAK becomes autophosphorylated and activates a number of downstream targets such as Src and PI3K. Src recruitment results in phosphorylation of several other residues associated with FAK, including paxillin [38]. Overexpression of FAK and PLC $\gamma$ in COS-7 cells increases PLC $\gamma$ enzymatic activity and tyrosine phosphorylation. FAK-induced PLC $\gamma$ phosphorylation may be due to FAK interaction and activation of Src family kinases [6]. Our results demonstrate that unlike Src, paxillin and PLC $\gamma$, which are highly phosphorylated in RA ST lining, only low numbers of RA ST lining cells are immunopositive for pFAK. pFAK staining in RA ST sublining was not greater than that in OA ST. Nevertheless, pFAK staining in RA ST lining and sublining was significantly higher than in ND STs. We additionally showed 


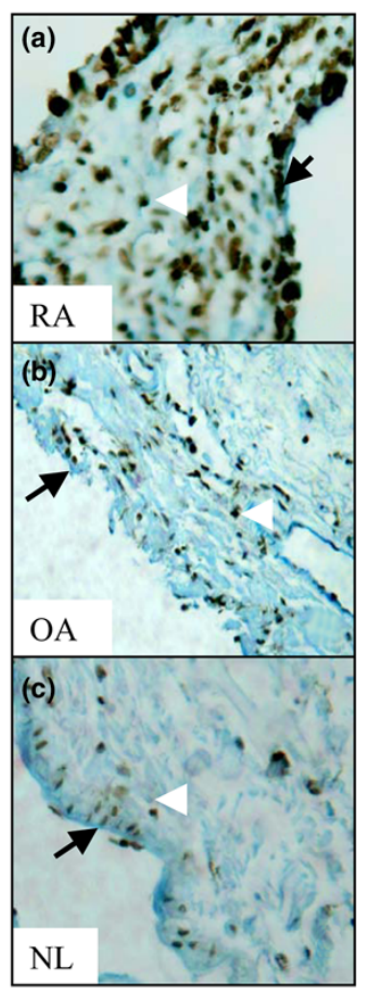

\section{(d) \\ RA ST $(n=9)$ \\ D $\square$ OA ST (n=8) \\ MV NL ST $(n=5)$}

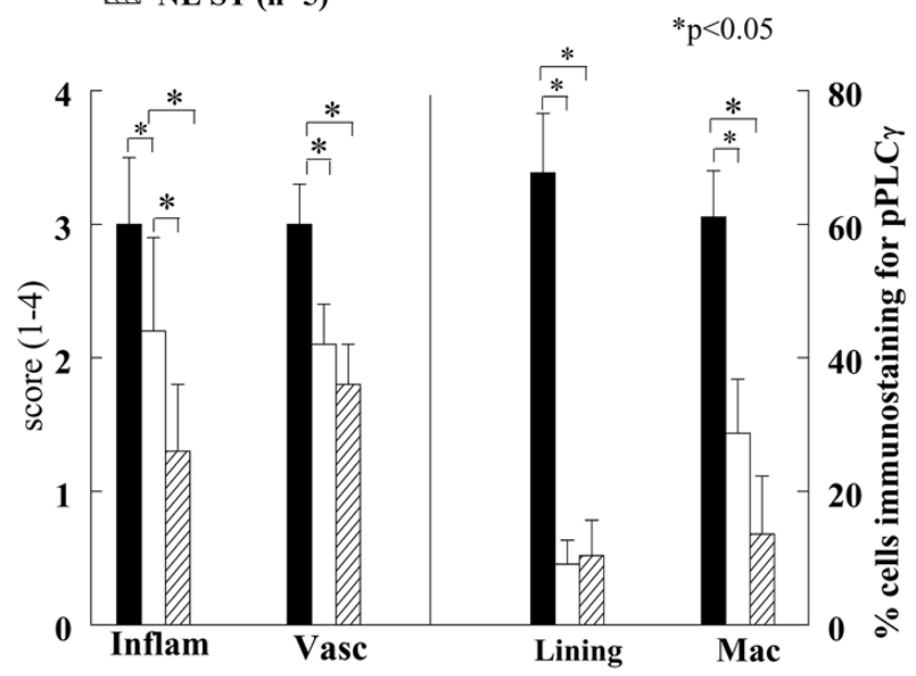

pPLC $\gamma$ immunostaining is higher in rheumatoid arthritis synovial tissue (RA ST) in comparison to osteoarthritis (OA) and normal donor (ND) ST. (a) RA ( $\times 200)$, compared to (b) OA ( $\times 200)$ and (c) ND (×200). (d) The quantification data obtained from a, b and c. Bars represent mean and SEM. Inflam, inflammatory score; Vasc, vascularity score; Lining, ST lining cell layer; Mac, subsynovial MФs.

that FAK, Pyk2 and Src were phosphorylated on unstimulated RA ST fibroblasts. The pro-inflammatory cytokines TNF $\alpha$ and IL1 $\beta$ further activated pFAK, pPyk2 and pSrc expression up to $120 \mathrm{~min}$. The activation of the FAK-Src-paxillin-PLC $\gamma$ pathway in RA ST lining and sublining suggests that these signaling proteins may be important for cell adhesion, cell migration and perhaps even $\mathrm{M} \Phi$ differentiation to osteoclasts.

MФs isolated from Pyk2-null mice showed impaired migration in response to chemokine stimulation. $\mathrm{Ca}^{2+}$ release in response to chemokine stimulation as well as integrin-mediated activation of PI3K were compromised in Pyk2-/- MФs [39]. These findings suggest that Pyk2 plays an important role in $\mathrm{M} \Phi$ function modulating migration [39]. Integrin av $\beta 3$-mediated signaling is dependent on the phosphorylation of Pyk2, Src, paxillin and PLC $\gamma$. However, M-CSF can induce osteoclast differentiation by recruiting Pyk2, PLC $\gamma$ and PI3K in a Src-independent manner, an effect which is blocked by PLC inhibitors [33]. These finding suggest that in the absence of one Pyk2 family member, other signaling proteins can rescue osteoclast function. Previous findings indicate that similar to FAK, Pyk2 is essential for integrin-mediated adhesion, monocyte-MФ migration, and osteoclast signaling and differentiation [18]. Our data demonstates that pPyk2, pSrc, pPaxillin and PPLC $\gamma$ are similarly expressed on RA ST lining and sublin- ing, and this expression is significantly higher than that found in OA and ND ST. The RA ST lining layer consists of fibroblasts and MФs that are in close proximity to one another. Although, pPyk2 is undetected on MФs or expressed in low levels on RA ST fibroblasts, the presence of TNF $\alpha$ and IL1 $\beta$ retains pPyk2 activation both in MФs and RA ST fibroblasts.

In RA ST fibroblasts, baseline levels of pSrc are greater than that of pFAK and pPyk2. Furthermore, TNF $\alpha$ and IL1 $\beta$ treatment increased pSrc expression modestly in RA ST fibroblasts. However, both cytokines induced pSrc levels to a greater extent in MФs. Src, paxillin and PLC $\gamma$ are associated with both integrin activation of FAK and Pyk2 pathways. It is controversial whether Src kinase activity is essential for Pyk2induced osteoclast bone resorption as Pyk2 can be phosphorylated in a $\mathrm{Ca}^{2+-}$-dependent manner [40]. In contrast, activation of Src is necessary for FAK function.

Circulating leukocytes, including lymphocytes and MФs, express high levels of paxillin [41]. Paxillin is a multi-domain adapter molecule which is important for recruiting multiple signaling protein to a specific location within the cell [42]. Paxillin is modulated in response to integrin-mediated cell adhesion and growth factors. In this study we demonstrate that pPaxillin is detected twofold higher in RA ST lining and sublining com- 


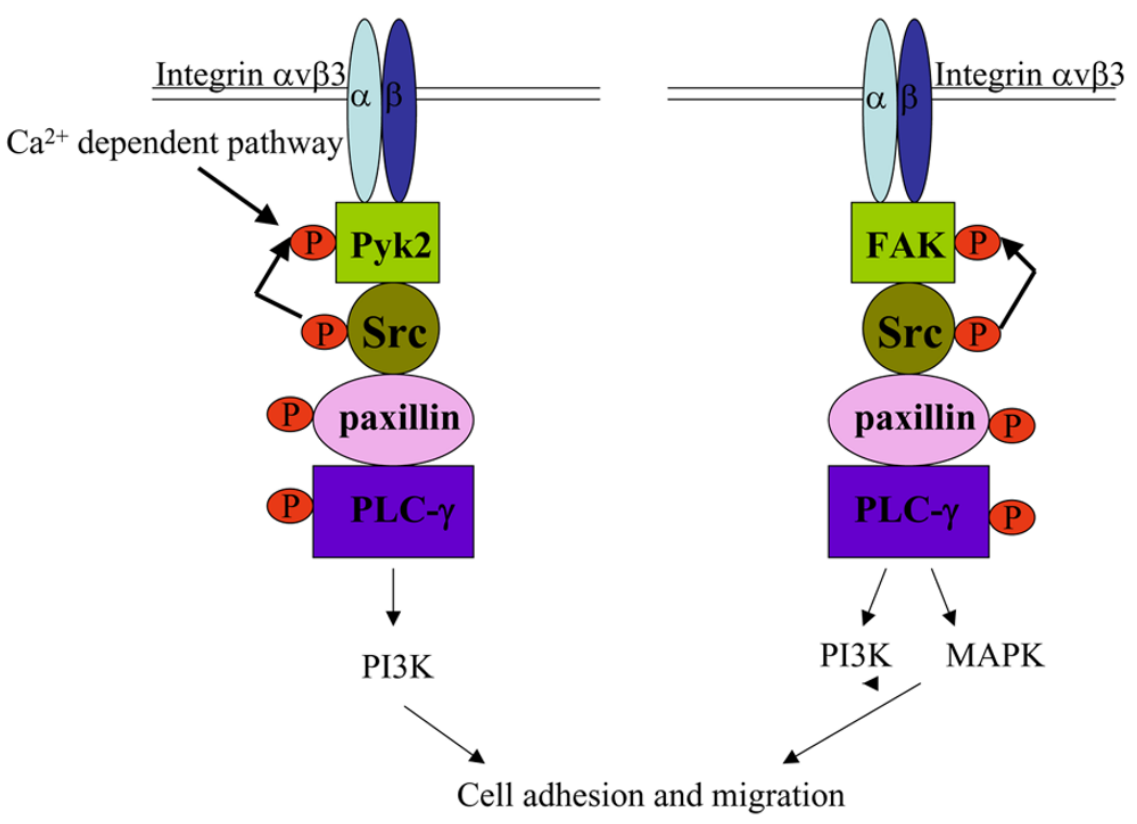

Putative integrin signaling pathways through Pyk2 or FAK. In response to integrin $\alpha v \beta 3$ activation, Pyk2 and/or FAK are recruited to a signaling complex that consists of Src, paxillin and PLC $\gamma$. Pyk2 may be phosphorylated through Src or other $\mathrm{Ca}^{2+}$ dependent pathways whereas FAK is phosphorylated through Src. Both Pyk2 and FAK can result in activation of PI3K and/or MAPK that may lead to cell adhesion and migration into the rheumatoid arthritis synovial tissue (RA ST).

pared to OA ST. The numbers of pPaxillin immunopositive cells are significantly lower in ND ST lining and sublining compared to RA ST, suggesting that activation of paxillin is associated with RA inflammation.

PLC $\gamma$-null fibroblasts are defective in adhesion, spreading and migration [43]. Integrin engagement by fibronectin induces tyrosine phosphorylation of PLC $\gamma 1$ and that this signaling event requires Src activity [43]. Further, paxillin associates with PLC $\gamma 1$ in cells grown on fibronectin that may be mediated by FAK or Pyk2 [44]. Although pPLC $\gamma$ is significantly upregulated on RA ST lining and sublining, it is similarly expressed in OA and ND ST.

Multiple signal-transduction pathways have been implicated in RA, most notably protein kinases such as MAPK [45] and PI3K [46]. Preclinical models have confirmed the therapeutic potential of p38 MAPK $[47,48]$ and PI3K [49] inhibition and clinical trials are under way to evaluate inhibitors for these signaling pathways [45]. Although the preliminary results obtained from animal models are promising, proof of safety has not yet been obtained. As both p38 MAPK and PI3K are involved in normal processes, inhibition of these signaling pathways may produce untoward effects. Hence, identifying the intermediary signaling proteins that are dysfunctional in RA and not in ND may offer new therapeutic options.

\section{Conclusion}

Taken together, our results demonstrate that FAK family kinases, including FAK and Pyk2, and their associated signaling intermediates, namely Src, paxillin and PLC $\gamma$ are phosphorylated in RA ST lining and sublining. Although both FAK and Pyk2 have been implicated in cell adhesion, migration and osteoclast differentiation, alternative pathways may be used for each function. Inhibiting activation of the FAK superfamily may suppress cell adhesion and migration into RA ST and provide a novel therapeutic target.

\section{List of abbreviations}

$\mathrm{AIA}=$ adjuvant induced arthritis; $F A K=$ focal adhesion kinase; $\mathrm{FGF}=$ fibroblast growth factor; IL = interleukin; $\mathrm{M}-\mathrm{CSF}=$ macrophage colony stimulating factor; $\mathrm{ND}=$ normal donor; $\mathrm{OA}=$ osteoarthritis; PB MФ = peripheral blood differentiated macrophages; PDGF = platelet derived growth factor; PLC = phospholipase $\mathrm{C} ; \mathrm{Pyk}=$ proline rich kinase; $\mathrm{RA}=$ rheumatoid arthritis; RANKL = receptor activator of NF-kappa B ligand; ST = synovial tissue; TBS-T = Tris-buffered saline Tween; TNF = tumor necrosis factor; VEGF = vascular endothelial growth factor.

\section{Competing interests}

The authors declare that they have no competing interests.

\section{Authors' contributions}

SS was responsible for design of the study, acquisition of data, analysis and interpretation of the data and manuscript 
preparation; HPR and GKH were responsible for acquisition of data; AEK was responsible for design of the study, interpretation of the data and manuscript preparation, and all authors have approved the content of the manuscript.

\section{Acknowledgements}

This work was supported by US National Institutes of Health grants AR049353, AR48267, HL58695, Al40987, The Gallagher Professorship for Arthritis Research, The Frederick G.L. Huetwell and William D. Robinson, M.D. Professor of Rheumatology, and funds from the Veteran's Administration Research Service.

\section{References}

1. Brown MC, Perrotta JA, Turner CE: Identification of LIM3 as the principal determinant of paxillin focal adhesion localization and characterization of a novel motif on paxillin directing vinculin and focal adhesion kinase binding. J Cell Biol 1996, 135:1109-1123.

2. Xing Z, Chen HC, Nowlen JK, Taylor SJ, Shalloway D, Guan JL: Direct interaction of v-Src with the focal adhesion kinase mediated by the Src SH2 domain. Mol Biol Cell 1994, 5:413-421.

3. Chen HC, Guan JL: Association of focal adhesion kinase with its potential substrate phosphatidylinositol 3-kinase. Proc Natl Acad Sci USA 1994, 91:10148-10152.

4. Hildebrand JD, Schaller MD, Parsons JT: Paxillin, a tyrosine phosphorylated focal adhesion-associated protein binds to the carboxyl terminal domain of focal adhesion kinase. Mol Biol Cell 1995, 6:637-647.

5. Polte TR, Hanks SK: Interaction between focal adhesion kinase and Crk-associated tyrosine kinase substrate p130Cas. Proc Natl Acad Sci USA 1995, 92:10678-10682.

6. Zhang X, Chattopadhyay A, Ji OS, Owen JD, Ruest PJ, Carpenter G, Hanks SK: Focal adhesion kinase promotes phospholipase C-gamma1 activity. Proc Natl Acad Sci USA 1999, 96:9021-9026.

7. Maru Y, Hanks SK, Shibuya M: The tubulogenic activity associated with an activated form of Flt-1 kinase is dependent on focal adhesion kinase. Biochim Biophys Acta 2001, 1540:147-153.

8. Matsumoto Y, Tanaka K, Hirata G, Hanada M, Matsuda S, Shuto T, Iwamoto $Y$ : Possible involvement of the vascular endothelial growth factor-FIt-1-focal adhesion kinase pathway in chemotaxis and the cell proliferation of osteoclast precursor cells in arthritic joints. J Immuno/ 2002, 168:5824-5831.

9. Gilmore AP, Romer LH: Inhibition of focal adhesion kinase (FAK) signaling in focal adhesions decreases cell motility and proliferation. Mol Biol Cell 1996, 7:1209-1224.

10. Chen HC, Appeddu PA, Parsons JT, Hildebrand JD, Schaller MD, Guan JL: Interaction of focal adhesion kinase with cytoskeletal protein talin. J Biol Chem 1995, 270:16995-16999.

11. Horwitz A, Duggan K, Buck C, Beckerle MC, Burridge K: Interaction of plasma membrane fibronectin receptor with talin - a transmembrane linkage. Nature 1986, 320:531-533.

12. Schaller MD, Hildebrand JD, Shannon JD, Fox JW, Vines RR, Parsons JT: Autophosphorylation of the focal adhesion kinase, pp125FAK, directs SH2-dependent binding of pp60src. Mol Cell Biol 1994, 14:1680-1688.

13. Chen HC, Appeddu PA, Isoda H, Guan JL: Phosphorylation of tyrosine 397 in focal adhesion kinase is required for binding phosphatidylinositol 3-kinase. J Biol Chem 1996, 271:26329-26334.

14. Schlaepfer DD, Hunter T: Focal adhesion kinase overexpression enhances ras-dependent integrin signaling to ERK2/ mitogen-activated protein kinase through interactions with and activation of c-Src. J Biol Chem 1997, 272:13189-13195.

15. Avraham H, Park SY, Schinkmann K, Avraham S: RAFTK/Pyk2mediated cellular signalling. Cell Signal 2000, 12:123-133.

16. Andreev J, Simon JP, Sabatini DD, Kam J, Plowman G, Randazzo $\mathrm{PA}$, Schlessinger J: Identification of a new Pyk2 target protein with Arf-GAP activity. Mol Cell Biol 1999, 19:2338-2350.
17. Schlaepfer DD, Hauck CR, Sieg DJ: Signaling through focal adhesion kinase. Prog Biophys Mol Biol 1999, 71:435-478.

18. Xiong WC, Feng X: PYK2 and FAK in osteoclasts. Front Biosci 2003, 8:d1219-d1226.

19. Duong LT, Lakkakorpi PT, Nakamura I, Machwate M, Nagy RM, Rodan GA: PYK2 in osteoclasts is an adhesion kinase, localized in the sealing zone, activated by ligation of alpha(v)beta3 integrin, and phosphorylated by src kinase. J Clin Invest 1998 102:881-892.

20. Nakagawa M, Kaneda T, Arakawa T, Morita S, Sato T, Yomada T, Hanada K, Kumegawa M, Hakeda Y: Vascular endothelial growth factor (VEGF) directly enhances osteoclastic bone resorption and survival of mature osteoclasts. FEBS Lett 2000, 473:161-164.

21. Itonaga I, Fujikawa $Y$, Sabokbar A, Murray DW, Athanasou NA: Rheumatoid arthritis synovial macrophage-osteoclast differentiation is osteoprotegerin ligand-dependent. J Patho/ 2000, 192:97-104.

22. Kharbanda S, Saleem A, Yuan Z, Emoto Y, Prasad KV, Kufe D: Stimulation of human monocytes with macrophage colonystimulating factor induces a Grb2-mediated association of the focal adhesion kinase pp125FAK and dynamin. Proc Natl Acad Sci USA 1995, 92:6132-6136.

23. Altman $R$, Asch $E$, Bloch D, Bole G, Borenstein D, Brandt $K$, Christy W, Cooke TD, Greenwald R, Hochberg M, et al.: Development of criteria for the classification and reporting of osteoarthritis. Classification of osteoarthritis of the knee. Arthritis Rheum 1986, 29:1039-1049.

24. Altman R, Alarcon G, Appelrouth D, Bloch D, Borenstein D, Brandt K, Brown C, Cooke TD, Daniel W, Feldman D, et al:: The American College of Rheumatology criteria for the classification and reporting of osteoarthritis of the hip. Arthritis Rheum 1991, 34:505-514.

25. Tokuhira M, Hosaka S, Volin MV, Haines GK 3rd, Katschke KJ Jr, Kim S, Koch AE: Soluble vascular cell adhesion molecule 1 mediation of monocyte chemotaxis in rheumatoid arthritis. Arthritis Rheum 2000, 43:1122-1133.

26. Shahrara S, Amin MA, Woods JM, Haines GK, Koch AE: Chemokine receptor expression and in vivo signaling pathways in the joints of rats with adjuvant-induced arthritis. Arthritis Rheum 2003, 48:3568-3583

27. Shahrara S, Proudfoot AE, Woods JM, Ruth JH, Amin MA, Park CC, Haas CS, Pope RM, Haines GK, Zha YY, et al:: Amelioration of rat adjuvant-induced arthritis by Met-RANTES. Arthritis Rheum 2005, 52:1907-1919.

28. Ruth JH, Shahrara S, Park CC, Morel JC, Kumar P, Oin S, Koch $\mathrm{AE}$ : Role of macrophage inflammatory protein-3alpha and its ligand CCR6 in rheumatoid arthritis. Lab Invest 2003, 83:579-588.

29. Shahrara S, Park CC, Temkin V, Jarvis JW, Volin MV, Pope RM: RANTES modulates TLR4-induced cytokine secretion in human peripheral blood monocytes. J Immunol 2006, 177:5077-5087.

30. Kohno M, Aikawa $Y$, Tsubouchi $Y$, Hashiramoto A, Yamada $R$, Kawahito Y, Inoue K, Kusaka Y, Kondo M, Sano H: Inhibitory effect of T-614 on tumor necrosis factor-alpha induced cytokine production and nuclear factor-kappaB activation in cultured human synovial cells. J Rheumatol 2001, 28:2591-2596.

31. Baig $S$, Patel $Y$, Coussons $P$, Grant R: Erythropoietin and interleukin-1beta modulate nitrite production in a Swiss 3T3 cell model of rheumatoid synovial fibroblasts. Biochem Soc Trans 2002, 30:883-886.

32. Miyazaki T, Tanaka S, Sanjay A, Baron R: The role of c-Src kinase in the regulation of osteoclast function. Mod Rheumato/ 2006, 16:68-74.

33. Nakamura I, Lipfert L, Rodan GA, Le TD: Convergence of alpha(v)beta(3) integrin- and macrophage colony stimulating factor-mediated signals on phospholipase Cgamma in prefusion osteoclasts. J Cell Biol 2001, 152:361-373.

34. Wang $\mathrm{Q}$, Xie $Y$, Du OS, Wu XJ, Feng X, Mei L, McDonald JM, Xiong WC: Regulation of the formation of osteoclastic actin rings by proline-rich tyrosine kinase 2 interacting with gelsolin. $J$ Cell Biol 2003, 160:565-575.

35. Zhang Z, Neff L, Bothwell AL, Baron R, Horne WC: Calcitonin induces dephosphorylation of Pyk2 and phosphorylation of focal adhesion kinase in osteoclasts. Bone 2002, 31:359-365. 
36. Tanaka S, Takahashi N, Udagawa N, Murakami H, Nakamura I, Kurokawa T, Suda T: Possible involvement of focal adhesion kinase, p125FAK, in osteoclastic bone resorption. J Cell Biochem 1995, 58:424-435.

37. Kim I, Kim HG, Moon SO, Chae SW, So JN, Koh KN, Ahn BC, Koh GY: Angiopoietin-1 induces endothelial cell sprouting through the activation of focal adhesion kinase and plasmin secretion. Circ Res 2000, 86:952-959.

38. Guan JL: Focal adhesion kinase in integrin signaling. Matrix Biol 1997, 16:195-200.

39. Okigaki M, Davis C, Falasca M, Harroch S, Felsenfeld DP, Sheetz MP, Schlessinger J: Pyk2 regulates multiple signaling events crucial for macrophage morphology and migration. Proc Natl Acad Sci USA 2003, 100:10740-10745.

40. Sanjay A, Houghton A, Neff L, DiDomenico E, Bardelay C, Antoine E, Levy J, Gailit J, Bowtell D, Horne WC, et al:: Cbl associates with Pyk2 and Src to regulate Src kinase activity, alpha(v)beta(3) integrin-mediated signaling, cell adhesion, and osteoclast motility. J Cell Biol 2001, 152:181-195.

41. Turner CE: Paxillin. Int J Biochem Cell Biol 1998, 30(9):955-959.

42. Turner CE, Miller JT: Primary sequence of paxillin contains putative $\mathrm{SH} 2$ and $\mathrm{SH} 3$ domain binding motifs and multiple LIM domains: identification of a vinculin and pp125Fak-binding region. J Cell Sci 1994, 107:1583-1591.

43. Tvorogov D, Wang XJ, Zent R, Carpenter G: Integrin-dependent PLC-gamma1 phosphorylation mediates fibronectin-dependent adhesion. J Cell Sci 2005, 118:601-610.

44. Chang JS, Iwashita S, Lee YH, Kim MJ, Ryu SH, Suh PG: Transformation of rat fibroblasts by phospholipase C-gamma1 overexpression is accompanied by tyrosine dephosphorylation of paxillin. FEBS Lett 1999, 460:161-165.

45. Sweeney SE, Firestein GS: Signal transduction in rheumatoid arthritis. Curr Opin Rheumatol 2004, 16:231-237.

46. Morel J, Berenbaum F: Signal transduction pathways: new targets for treating rheumatoid arthritis. Joint Bone Spine 2004, 71:503-510.

47. Badger AM, Griswold DE, Kapadia R, Blake S, Swift BA, Hoffman SJ, Stroup GB, Webb E, Rieman DJ, Gowen M, et al.: Diseasemodifying activity of SB 24 a selective inhibitor of p38 mitogen-activated protein kinase, in rat adjuvant-induced arthritis. Arthritis Rheum 2000, 43:175-183.

48. Nishikawa M, Myoui A, Tomita T, Takahi K, Nampei A, Yoshikawa $\mathrm{H}$ : Prevention of the onset and progression of collageninduced arthritis in rats by the potent 038 mitogen-activated protein kinase inhibitor FR167653. Arthritis Rheum 2003, 48:2670-2681

49. Camps M, Ruckle T, Ji H, Ardissone V, Rintelen F, Shaw J, Ferrandi C, Chabert C, Gillieron C, Francon B, et al:: Blockade of PI3Kgamma suppresses joint inflammation and damage in mouse models of rheumatoid arthritis. Nat Med 2005, 11:936-943. 